Supporting Information

\title{
Interfacial Adsorption of a Monoclonal Antibody and its Fab and Fc Fragments at the Oil/Water Interface
}

Sean Ruane ${ }^{1}$, Zongyi $\mathrm{Li}^{1}$, Mario Campana ${ }^{2}$, Xuzhi Hu${ }^{1}$, Haoning Gong ${ }^{1}$, John R. P. Webster ${ }^{2}$, Faisal Uddin ${ }^{3}$, Cavan Kalonia ${ }^{4}$, Steven M. Bishop ${ }^{4}$, Christopher F. van der Walle ${ }^{3}$, Jian R. Lu ${ }^{1 *}$

${ }^{1}$ Biological Physics Laboratory, School of Physics and Astronomy, University of Manchester, Oxford Road, Schuster Building, Manchester M13 9PL, UK.

${ }^{2}$ ISIS Neutron Facility, STFC, Chilton, Didcot OX11 0QZ, UK.

${ }^{3}$ Dosage Form Design \& Development, AstraZeneca, Granta Park, Cambridge CB21 6GH, UK

${ }^{4}$ Dosage Form Design \& Development, AstraZeneca, Gaithersburg, MD 20878, USA.

*Corresponding author: Jian R Lu (email: j.lu@ manchester.ac.uk; Tel: +44 161 2003926) 


\section{S.1 Fitting Parameters}

Table S1 shows the parameters used for the fitting of neutron reflectivity data for each protein in the three aqueous contrasts used. Protein densities and resulting adsorbed masses are given in $\mathrm{H}_{2} \mathrm{O}$.

\begin{tabular}{|l|l|l|l|l|l|l|}
\multicolumn{2}{l}{ Component } & \multicolumn{1}{l}{$\begin{array}{l}\text { Contrast } \\
\text { buffer }\end{array}$} \\
\cline { 2 - 7 } & CM Sapphire & 5526 & 3.16 & 175139 & 146,478 & \\
\hline COE-03 & CM Protein & 4440 & 2.58 & & 145,499 & \\
\hline & $\mathrm{H}_{2} \mathrm{O}$ & 3336 & 1.91 & & 144,505 & 1.37 \\
\hline Fc & CM Sapphire & 1855 & 3.05 & 60852 & 50,608 & \\
\hline & CM Protein & 1502 & 2.52 & & 50,326 & \\
\hline & $\mathrm{H}_{2} \mathrm{O}$ & 1143 & 1.88 & & 49,958 & 1.36 \\
\hline Fab & CM Sapphire & 1835 & 3.21 & 57173 & 47,955 & \\
\hline & CM Protein & 1468 & 2.61 & & 47,852 & \\
\hline & $\mathrm{H}_{2} \mathrm{O}$ & 1096 & 1.92 & & 47,291 & 1.37 \\
\hline
\end{tabular}

Table S1. The fitting parameters used for each contrast and protein. *nonglycosylated 

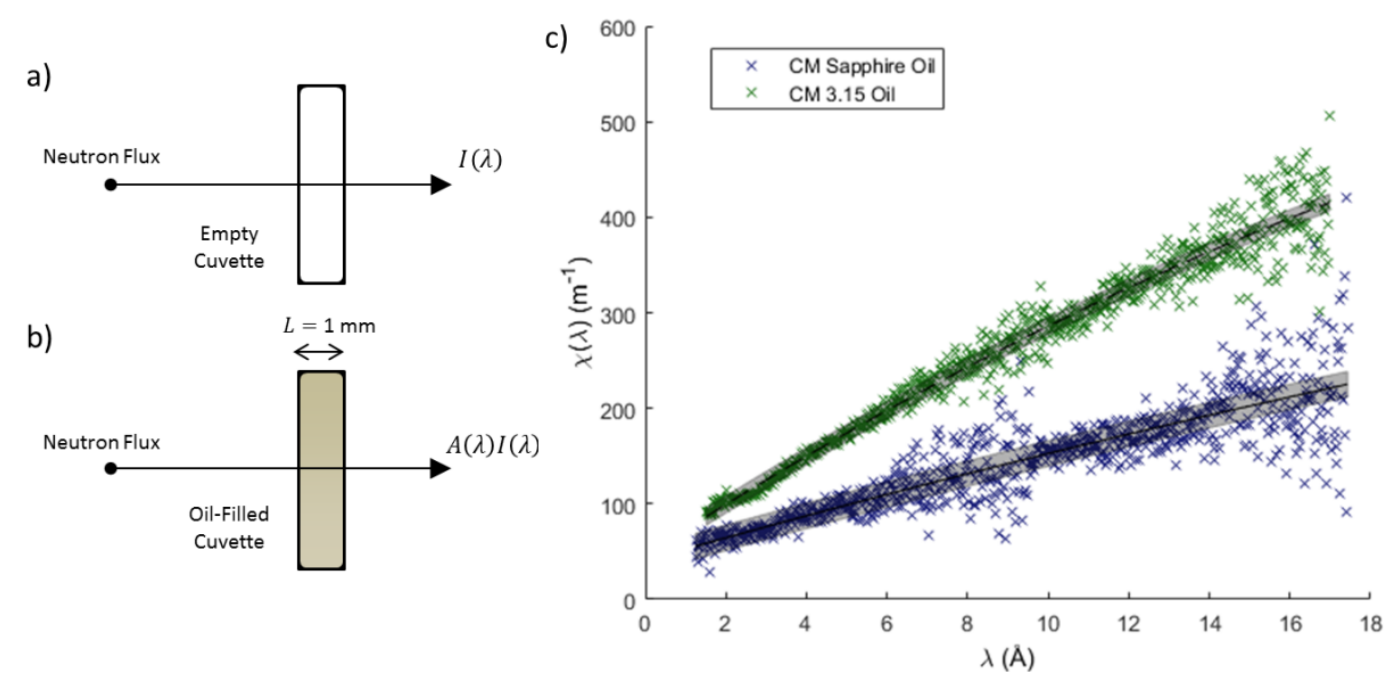

Fig S1. The direct neutron beam passing through an empty and oil-filled cuvette respectively (a and b). Plot of $\chi(\lambda)$ for oil CM to sapphire (SLD $5.65 \times 10^{-6} \AA^{-2}$ ) and CM 3.15 oil (SLD $3.15 \times 10^{-6} \AA^{-2}$ ) and best fitted polynomials (c).

The "straight-through" method is used measure to $\chi(\lambda)$, the attenuation per unit length of the oil. The neutron beam is directed straight through a cuvette of known thickness, in this case 1 $\mathrm{mm}$, with no reflection. The intensity of the resulting beam is measured as a function of $\lambda$. This intensity function is then normalised using the intensity through an empty cuvette, eliminating all factors except the oil attenuation $A(\lambda)$, which can be used to find $\chi(\lambda)$ via equation 5 in the main text. $\chi(\lambda)$ was then fitted with a $3^{\text {rd }}$ order polynomial for data analysis purposes, the best fit values are shown in Table S2. This method was used to determine $\chi(\lambda)$ for oil CM to sapphire (SLD 5.65×10 $\left.0^{-6} \AA^{-2}\right)$ and CM 3.15 oil $\left(3.15 \times 10^{-6} \AA^{-2}\right)$.

\begin{tabular}{|cc|ccc|}
\multicolumn{1}{c|}{ Oil } & SLD $\left(\AA^{-2}\right)$ & $\mathrm{A}\left(\mathrm{m}^{-4}\right)$ & $\mathrm{B}\left(\mathrm{m}^{-3}\right)$ & $\mathrm{C}\left(\mathrm{m}^{-2}\right)$ \\
\hline $\begin{array}{c}\text { CM } \\
\text { Sapphire }\end{array}$ & $5.65 \times 10^{-6}$ & -0.16 & 23.99 & 82.03 \\
\hline CM 3.15 & $3.15 \times 10^{-6}$ & -0.64 & 54.35 & 92.97 \\
\hline
\end{tabular}

Table S2. Values for the polynomial fits of $\chi(\lambda)$ in the format $\chi(\lambda)=A \lambda^{3}+B \lambda^{2}+C \lambda$. 


\section{S.3 "Double Critical-Edge" Thickness Measurements}

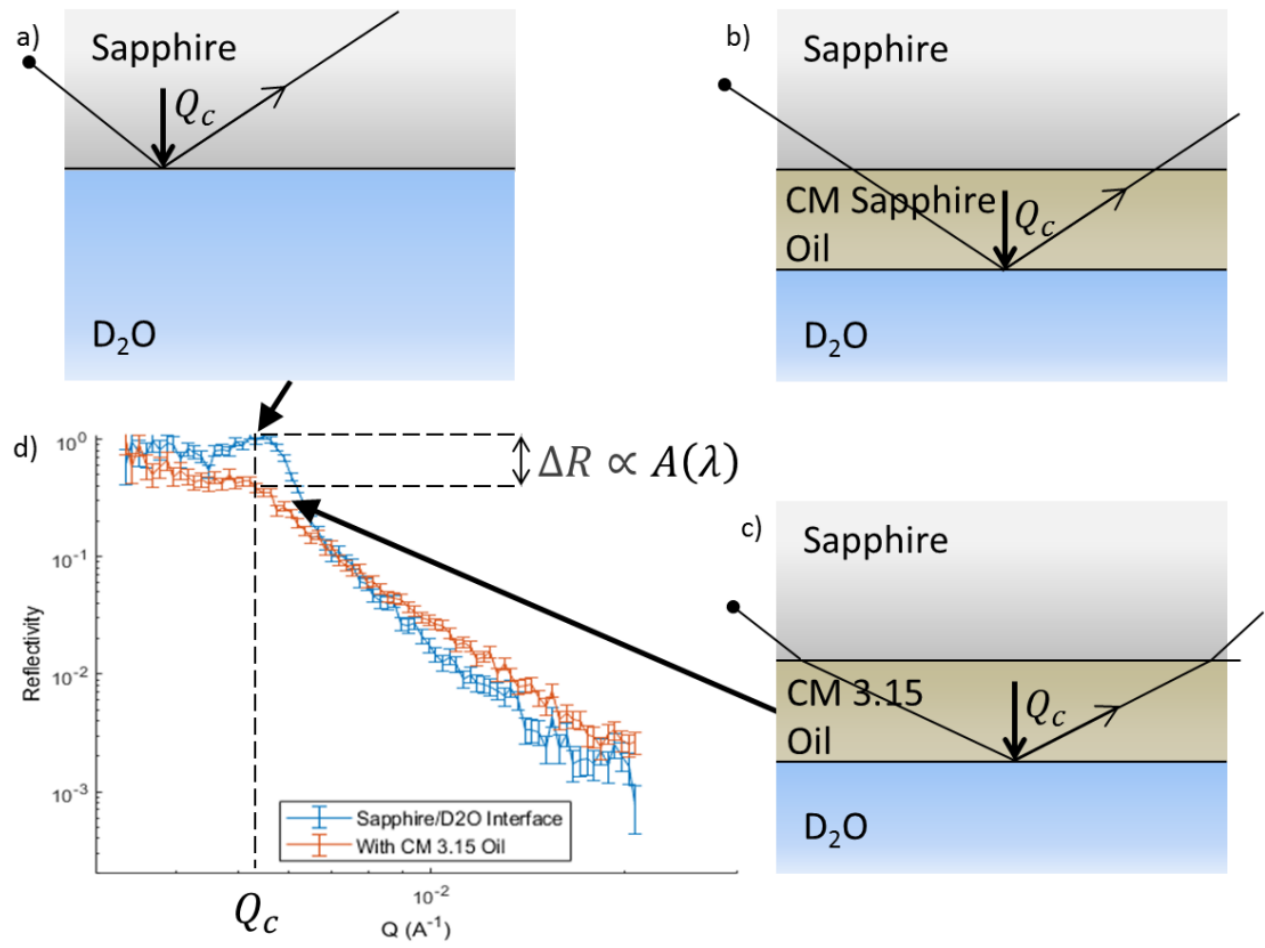

Fig S2. Schematic of the thickness of the layers determined by the "Double Critical-Edge" method. a) shows the reflection from the substrate, $b$ ) shows the reflection with oil contrast matched to the sapphire layer, c) shows an exaggerated example of the additional refraction effect caused by a noncontrast matched oil, and d) shows reflectivity data demonstrating this double critical edge effect.

Figure S2 shows how the double critical edge method was used to determine the attenuation of the layer, which, with a known $\chi(\lambda)$, can be used to determine the layer thickness. Figure S2a shows the total internal reflection from the substrate, below the critical angle, this measurement is used to give an absolute scaling value for effect. Figure S2b shows that when an oil film contrast matched to sapphire is added, the total internal reflection occurs from the oil/water interface, with the beam passing through the oil layer. If oil with a lower SLD is added, as shown in Figure S2c, there is a slight refractive effect, decreasing the effective angle and therefore increasing the path length through the oil. However, as the change in neutron refractive index of materials is extremely low relative to, for example, photons, this change in angle is extremely small, and therefore the resulting change in the path length is negligible.

The measurements used to determine the thickness, with an example displayed in Figure S2d, used CM 3.15 (SLD $3.15 \times 10^{-6} \AA^{-2}$ ) oil, with a higher proportion of h-hexadecane than oil CM to sapphire, and therefore greater $\chi(\lambda)$. This gives a larger attenuation, increasing the difference between the un-attenuated and attenuated critical edges (and therefore the signal-toerror ratio), allowing more accurate measurements of the thickness. 


\section{S.4 Modelling Globular Penetration}

Table S3 demonstrates the best fitted thicknesses modelled for globular protein penetrating into the oil interface. As shown in Figure S3, this model treats the volume fraction of the protein as consistent in both oil and water layers, and varies the overall protein layer thickness and the depth of the oil-hydrated layer. More complex models allowing variation of volume fraction or three-phase oil-water-protein mixing were also used, and found similar results. At lower concentrations, the penetration into the interface appears to increase. This appears counterintuitive, as one might expect the amount of protein mixing to increase with concentration. However, this is due to the increased uncertainty in the penetration, as a smaller amount of total protein in the layer means that the same depth of penetration into the oil would result in lower total oil-protein mixing, and thus a smaller measurable effect on the $\mathrm{H}_{2} \mathrm{O}$ and CM protein buffers. The measurements with the highest adsorbed masses, which show little penetration, are therefore the most sensitive.

\begin{tabular}{|c|ccc|ccc|ccc|}
$\begin{array}{c}\text { Concentration } \\
(\mathrm{ppm})\end{array}$ & \multicolumn{2}{c}{ COE-3 } & \multicolumn{2}{c}{ Fab } & \multicolumn{2}{c}{ Fc } \\
\hline 10 & 0.5 & \pm & 2 & 3 & \pm & 4.5 & 2 & \pm & 3.5 \\
50 & 0.5 & \pm & 1.5 & 3.5 & \pm & 4.5 & 2 & \pm & 3 \\
200 & 0.5 & \pm & 0.5 & 0.5 & \pm & 2.5 & 0.5 & \pm & 1.5 \\
\hline
\end{tabular}

Table S3. Fitted parameters for protein penetration into the oil assuming adsorption from globular Fc, Fab and COE-3.

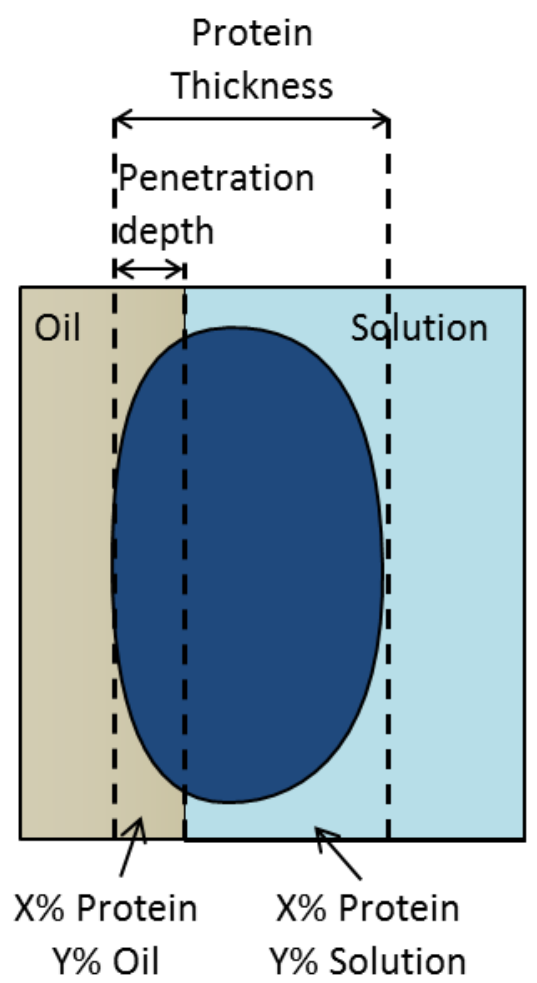

Fig S3. Schematic of the parameters of the globular penetration model, where $X+Y=100$. 

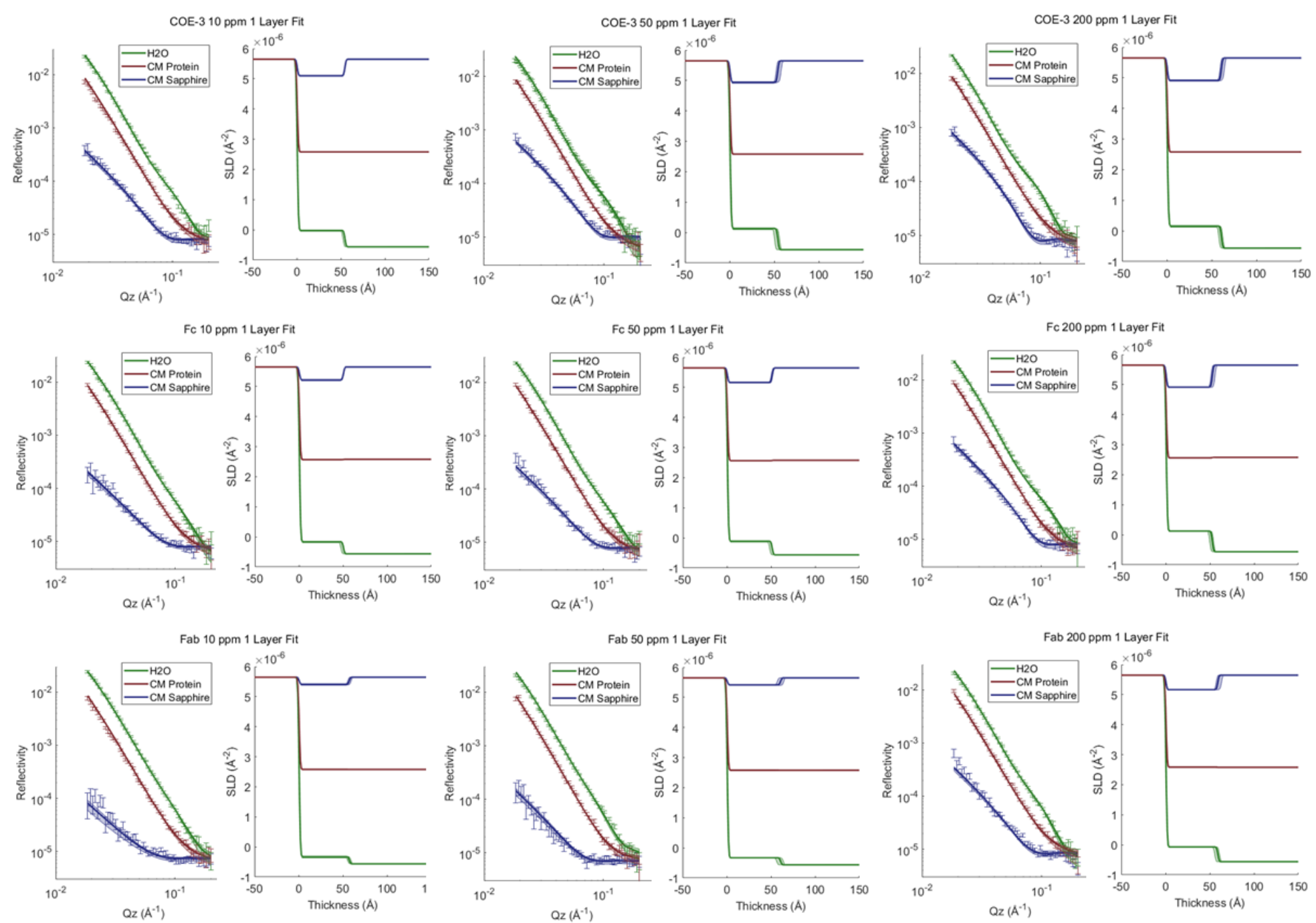

Fig S4. Fits to measured reflectivity profiles and SLD profiles for COE-3, Fab and Fc using a onelaver model.

\section{S.5 Fitted Reflectivity Data}

\begin{tabular}{|c|c|c|c|c|c|c|c|c|c|c|}
\hline \multirow{4}{*}{$\begin{array}{l}\text { Protein } \\
\text { COE-3 }\end{array}$} & $\begin{array}{l}\text { Concentration } \\
(\mathrm{ppm})\end{array}$ & \multicolumn{2}{|c|}{$\begin{array}{c}\text { Layer } \\
\text { Thickness } \\
(\AA)\end{array}$} & \multicolumn{3}{|c|}{$\varphi$ prot } & \multicolumn{3}{|c|}{$\Gamma(\mathrm{mg} / \mathrm{ml})$} & $x^{2}$ \\
\hline & 10 & 54 & \pm 1 & 0.22 & \pm & 0.01 & 1.61 & \pm & 0.06 & 9.56 \\
\hline & 50 & 54 & \pm 2 & 0.28 & \pm & 0.02 & 2.06 & \pm & 0.05 & 9.24 \\
\hline & 200 & 60 & \pm 2 & 0.29 & \pm & 0.02 & 2.40 & \pm & 0.06 & 11.94 \\
\hline \multirow{3}{*}{$\mathrm{Fab}$} & 10 & 57 & \pm 2 & 0.10 & \pm & 0.01 & 0.73 & \pm & 0.07 & 6.34 \\
\hline & 50 & 59 & \pm 1 & 0.10 & \pm & 0.01 & 0.78 & \pm & 0.06 & 5.75 \\
\hline & 200 & 59 & \pm 2 & 0.20 & \pm & 0.01 & 1.58 & \pm & 0.08 & 8.41 \\
\hline \multirow{3}{*}{$\mathrm{Fc}$} & 10 & & \pm 1 & 0.16 & \pm & 0.01 & 1.12 & \pm & 0.06 & 6.33 \\
\hline & 50 & 50 & \pm 1 & 0.19 & \pm & 0.01 & 1.29 & \pm & 0.04 & 6.52 \\
\hline & 200 & & \pm 2 & 0.28 & \pm & 0.01 & & \pm & 0.09 & 9.16 \\
\hline
\end{tabular}

Table S4. Fitted parameters and total adsorbed mass for the one-layer model with their respective errors and the goodness of fit in $\chi^{2}$. Note that the proportional error in the adsorbed mass is in some cases lower than the results of simple error propagation. This is as the Bayesian analysis accounts for the fact that layer thickness and volume fraction (and their corresponding errors) are anti-correlated, resulting in a more consistent mass value. 

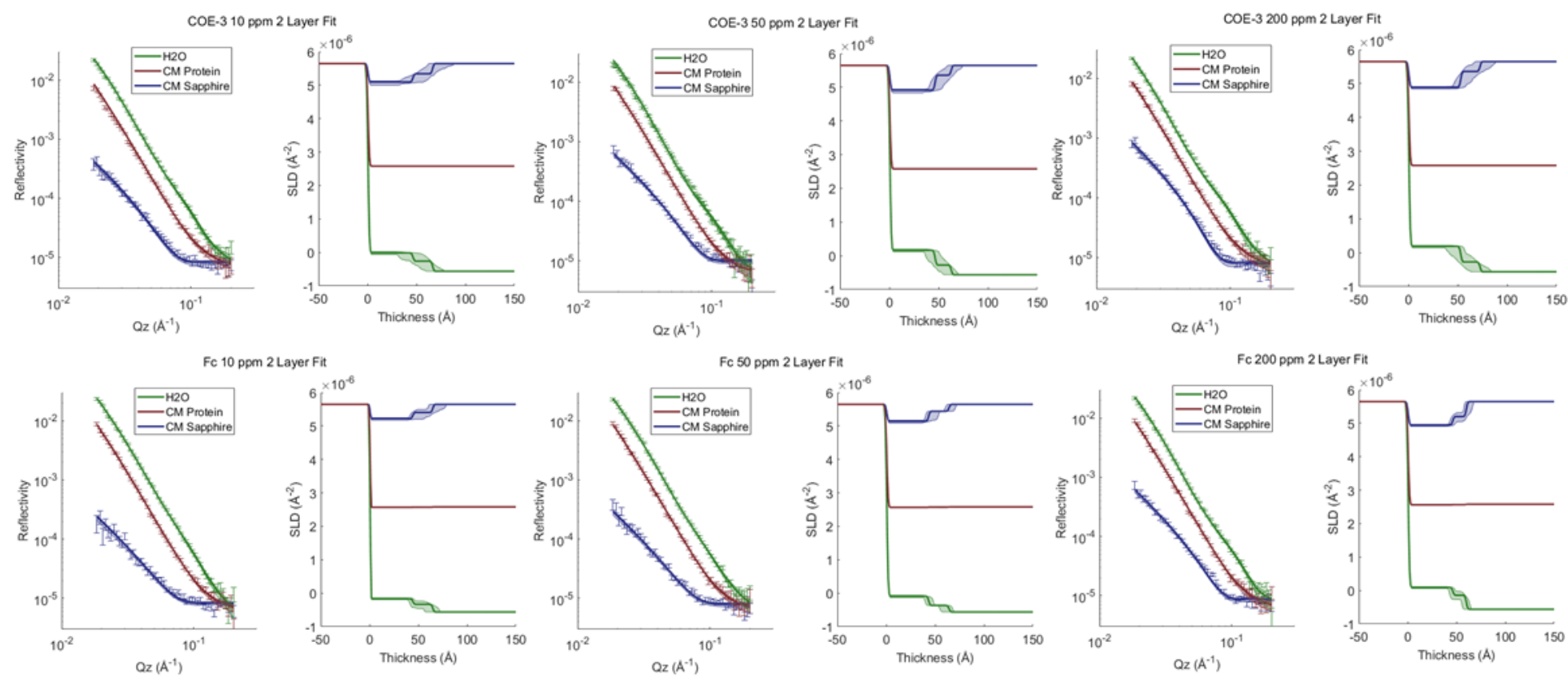

Fig S5. Fits to the reflectivity data, and SLD profiles for COE-3 and Fc using a two-layer model.

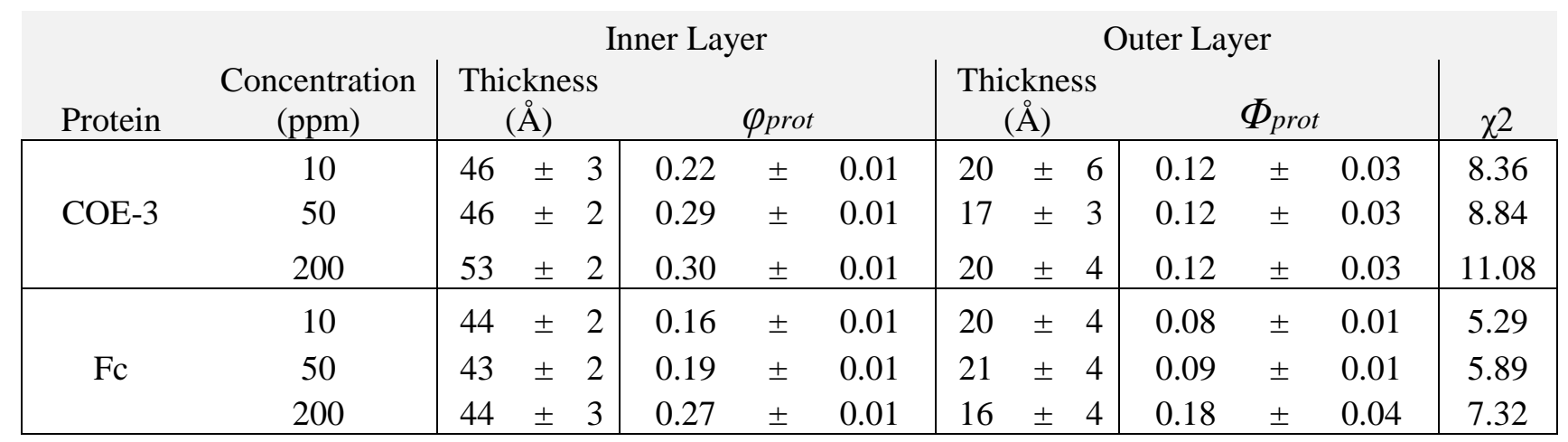

Table S5. Fitted parameters for the two-layer model with their respective errors and goodness of fit. 


\section{S.5 Area per Molecule}

Figure S6 shows the calculated Area Per Molecule (APM) for COE-3 and its Fab and Fc fragments plotted against concentration. These values assume a one-layer coverage.

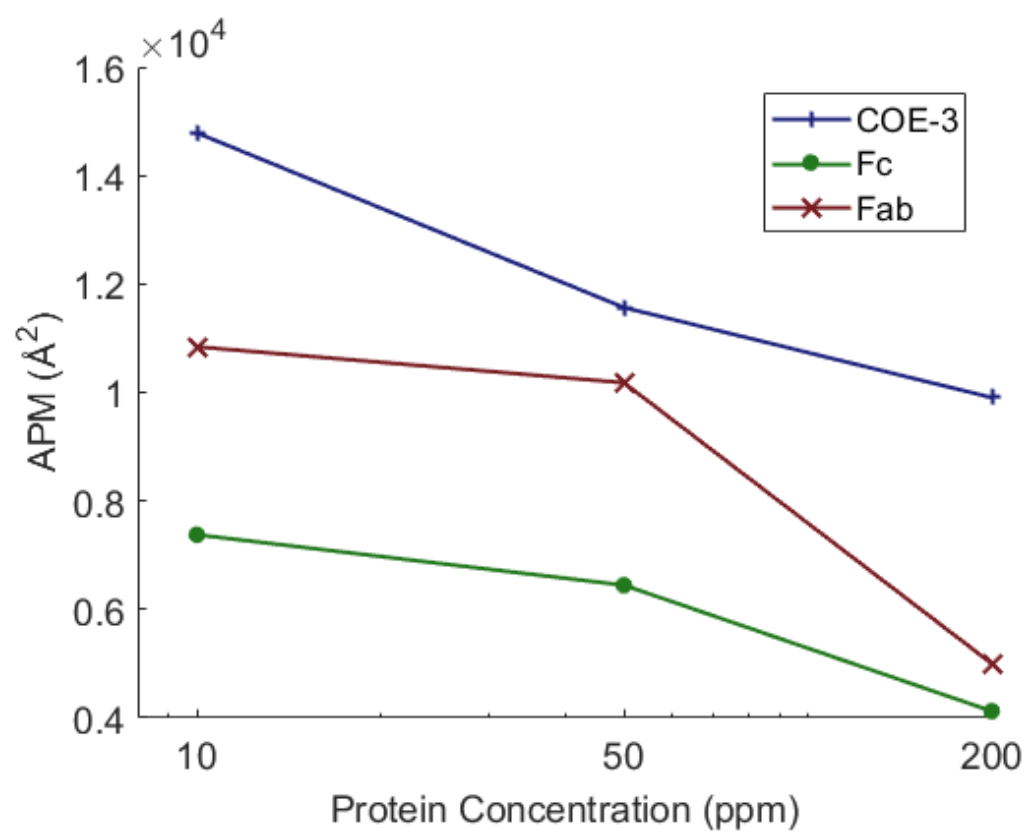

Fig S6. Area per molecule for COE-3, Fab and Fc plotted against concentration. 


\section{S.6 Oil/Water Interfacial Tensiometry}

Surface tension measurements were taken using a Krüss K11 surface tensiometer, adopting the Du Noüy ring method in a "pull" configuration. The protein solution is prepared in a clean borosilicate vessel. Then a platinum ring connected to a force meter is lowered into the solution. Purified hexadecane is then pipetted carefully onto the surface, creating an oil/water interface. The ring is then pulled into the interface, and the force measured. At the oil/water interface, the surface tension $\gamma$ is given by the equation:

$$
\gamma=\frac{F}{4 \pi R} f
$$

where $R$ is the radius of the ring, $f$ is the Huh and Mason correction factor ${ }^{\mathrm{S} 1}$ and $F$ is the maximum pulling force acting on the ring at equilibrium. All measurements were taken at $21 \pm 1^{\circ} \mathrm{C}$.
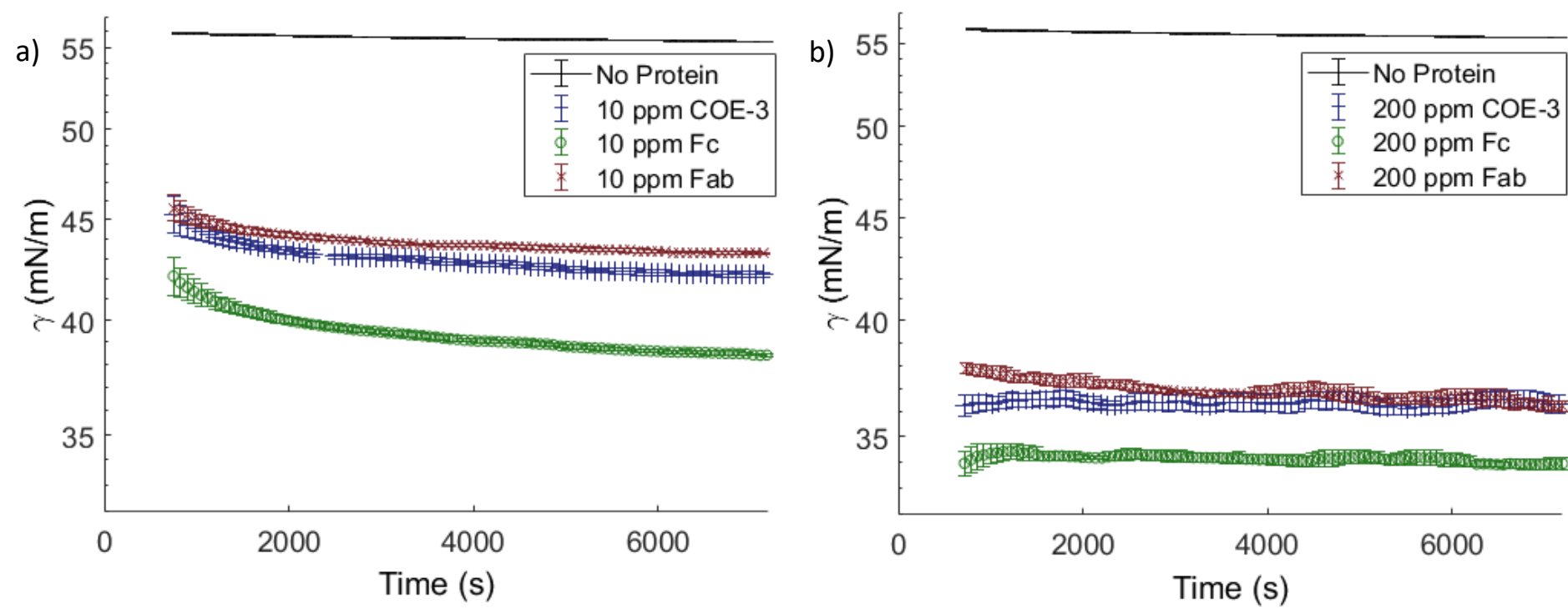

Fig S7. Interfacial tension measurements for COE-3 and its fragments at a) 10ppm and b) 200ppm concentration. All measurements taken in $\mathrm{pH} 5.525 \mathrm{mM}$ ionic strength Histidine buffer.

Figure S7 shows oil/water interfacial tension measurements for COE-3 and its fragments at 10 ppm (Figure S7a) and 200 ppm (Figure S7b). Points are calculated by the average from 10 moving measurements, with the error calculated as the standard deviation of these 10 points. The first 10 measurements have been excluded. Measurements without protein are in excellent agreement with published values for interfacial tension of the purified hexadecane/water interface $(55.2 \mathrm{mN} / \mathrm{m}) .{ }^{\mathrm{S} 2}$ None of these measurements results in interfacial tensions below 30 
$\mathrm{mN} / \mathrm{m}$. Low interfacial tension values can result in large-scale roughness due to capillary waves, thermal disruptions of the interfacial boundary, but at these relatively high interfacial tension values, this effect should be relatively insignificant. ${ }^{\mathrm{S3}}$

For COE-3 and Fc at 200 ppm, the interfacial tension reached equilibrium rather quickly (within $\sim 30 \mathrm{~min}$ ) and only slightly changed thereafter. This suggests that there were no significant long-term changes in protein structure, which would usually result in a notable decrease in surface tension, due to protein structural rearrangements producing a greater contact with the oil. The measurement for the Fc at 10ppm appears to show a slight long term decrease in interfacial tension but the difference is very small over the two hour period $(\sim 1.5$ $\mathrm{mN} / \mathrm{m}$ ) suggesting relatively small, if any, structural rearrangements. Similarly, for $10 \mathrm{ppm}$ Fab and COE-3 there was little change in surface tension after $30 \mathrm{~min}$. These tensiometry data inform us that, for the NR measurements, allowing the interfaces to equilibrate for $>30 \mathrm{~min}$ minimized the risk of significant changes occurring at the interface during the $2 \mathrm{hr} N R$ measurement period.

Consistent with the NR data of the adsorbed amount in $\mathrm{nmol} / \mathrm{m}^{2}$ (Figure 7), adsorption of Fc lowered the interfacial tension by the greatest amount. While the interfacial tensions for Fab and COE-3 were similar, the adsorbed amount of $\mathrm{Fab}$ in $\mathrm{nmol} / \mathrm{m}^{2}$ was higher than that of the whole mAb.

\section{References}

(S1) Huh, C.; Mason, S. G. A Rigorous Theory of Ring Tensiometry. Colloid \& Polymer Sci 1975, 253 (7), 566-580. https://doi.org/10.1007/BF01753960.

(S2) Goebel, A.; Lunkenheimer, K. Interfacial Tension of the Water/ $n$-Alkane Interface. Langmuir 1997, 13 (2), 369-372. https://doi.org/10.1021/la960800g.

(S3) Langevin, D. Capillary-Wave Techniques for the Measurement of Surface Tension and Surface Viscoelasticity. Colloids and Surfaces 1990, 43 (2), 121-131. https://doi.org/10.1016/0166-6622(90)80284-B. 